\title{
An In Vitro-In Vivo Evaluation of the Antiproliferative and Antiangiogenic Effect of Flavone Apigenin against SK-MEL-24 Human Melanoma Cell Line
}

\author{
Alexandra Ghitu, ${ }^{1,2}$ Ioana Zinuca Pavel $\mathbb{D}^{1,2}$ Stefana Avram ${ }^{1 D},{ }^{1,2}$ Brigitta Kis, ${ }^{1}$ \\ Daliana Minda, ${ }^{1,2}$ Cristina Adriana Dehelean, ${ }^{2,3}$ Valentina Buda, ${ }^{2,4}$ Roxana Folescu, ${ }^{5}$ \\ and Corina Danciu ${ }^{1,2}{ }^{1,2}$
${ }^{1}$ Department of Pharmacognosy, "Victor Babeș” University of Medicine and Pharmacy Timișoara, Romania, Eftimie Murgu Sq. No. 2, 300041 Timişoara, Romania
${ }^{2}$ Research Center for Pharmaco-Toxicological Evaluation, "Victor Babeș” University of Medicine and Pharmacy Timișoara, Romania, Eftimie Murgu Sq. No. 2, 300041 Timișoara, Romania
${ }^{3}$ Department of Toxicology, "Victor Babeș” University of Medicine and Pharmacy Timișoara, Romania, Eftimie Murgu Sq. No. 2, 300041 Timișoara, Romania
${ }^{4}$ Department of Pharmacology and Clinical Pharmacy, "Victor Babeș" University of Medicine and Pharmacy Timişoara, Romania, Eftimie Murgu Sq. No. 2, 300041 Timișoara, Romania
${ }^{5}$ Department of Balneology, Medical Recovery and Rheumatology, "Victor Babeș" University of Medicine and Pharmacy Timișoara, \\ Romania, Eftimie Murgu Sq. No. 2, 300041 Timișoara, Romania
}

Correspondence should be addressed to Ioana Zinuca Pavel; ioanaz.pavel@umft.ro

Received 27 January 2021; Revised 9 April 2021; Accepted 5 June 2021; Published 22 June 2021

Academic Editor: Elena Andreucci

\begin{abstract}
Copyright (c) 2021 Alexandra Ghitu et al. This is an open access article distributed under the Creative Commons Attribution License, which permits unrestricted use, distribution, and reproduction in any medium, provided the original work is properly cited.

One of the most important class of natural compounds with successful preclinical results in the management of cancer is the flavonoids. Due to the plethora of biological activities, apigenin $\left(4^{\prime}, 5,7\right.$ trihydroxyflavone $)$ is a main representant of the flavone subclass. Although the antiproliferative and antiangiogenic effects of apigenin were studied on a significant number of human and murine melanoma cell lines, in order to complete the data existing in the literature, the aim of this study is to evaluate the in vitro effect of apigenin on SK-MEL-24 human melanoma cell line as well as in vivo on tumor angiogenesis using the aforementioned cell line on the chorioallantoic membrane assay. Results have shown that in the range of tested doses, the phytocompound presents significant antiproliferative, cytotoxic, and antimigratory potential at $30 \mu \mathrm{M}$, respectively, $60 \mu \mathrm{M}$. Moreover, the phytocompound in both tested concentrations limited melanoma cell growth and migration and induced a reduced angiogenic reaction limiting melanoma cell development.
\end{abstract}

\section{Introduction}

From the earliest times, different types of plant extracts represented an important option for the treatment and/or prevention of a wide range of pathologies. From the past to the present, herbs have been used as teas, powders, different types of extracts, or purified active phytocompounds included in various pharmaceutical formulations [1].
According to World Health Organization, about $88 \%$ of member states are using traditional and alternative medicine [2]. Recently, modern techniques have allowed the isolation and/or synthesis/semisynthesis in the laboratory of a large number of pharmacologically active phytocompounds. The benefits of natural molecules are known from centuries, and this approach is expanding because of the significant capacity of phytocompounds-alone or in a synergistic way 
as found in extracts-to prevent or to treat different pathologies. To this aspect is added the fact that administered correctly, respecting the doses and the time interval, the vast majority of natural compounds have limited side effects. Up to date the main focus of the researchers is to identify and to establish the bioactive compounds' chemical structure and also their mechanism of action and toxicological impact [3] Since Plant Kingdom is an inexhaustible source of new bioactive molecules, an increased number of new molecules used in current well-established protocols in medicine are provided from natural compounds [1].

The variety of bioactive compounds is represented by different classes of phytocompounds including polyphenols, flavonoids, anthocyanins, saponins, triterpenes, tannins, alkaloids, glycosides, gums, resins, and volatile oils [4].

Many of these classes of phytochemicals have demonstrated to possess in vitro antiproliferative, proapoptotic, cytotoxic activity on different cancer cell lines, or/and anticancer activity in different experimental animal models [59]. Moreover, clinical trials that evaluate natural compounds have been conducted and are in progress [10-16].

One of the most important class of natural compounds with successful preclinical results in the management of cancer is the flavonoids. This polyphenolic family has a common structure of phenyl-benzo-piran, and depending on the oxidation degree, the nature of the substituents attached to $\mathrm{C} 2 / \mathrm{C} 3$, and the hydroxylation pattern of the nucleus, the flavonoids are divided into different subclasses, of which the most important are flavanols, flavanones, flavonols, flavones, isoflavones, anthocyanidins, chalcones, aurones, and catechines [17].

The main interest of this current study is on the flavone apigenin (API), also known as $4^{\prime}, 5,7$ trihydroxyflavone and characterized by $\mathrm{C}_{15} \mathrm{H}_{10} \mathrm{O}_{5}$ chemical structure. API is well distributed in a variety of fruits, vegetables, and medicinal plants. Significant sources of API can be found in chamomile, parsley, celery, spices, thyme, onion, tea, and wine [5].

Studies showed that the richest sources of API can be detected in dried chamomile $(3,000-5,000 \mu \mathrm{g} / \mathrm{g})$, parsley $(2,154.6 \mu \mathrm{g} / \mathrm{g})$, celery seed $(786.5 \mu \mathrm{g} / \mathrm{g})$, Chinese celery $(240$ $\mu \mathrm{g} / \mathrm{g})$, kumquats $(218.7 \mu \mathrm{g} / \mathrm{g})$, celery $(191 \mu \mathrm{g} / \mathrm{g})$, vine spinach $(622 \mu \mathrm{g} / \mathrm{g})$, Mexican oregano $(177.1 \mu \mathrm{g} / \mathrm{g})$, artichoke $(74.8$ $\mu \mathrm{g} / \mathrm{g})$, juniper berries $(72.6 \mu \mathrm{g} / \mathrm{g})$, and peppermint $(53.9$ $\mu \mathrm{g} / \mathrm{g})[18]$.

API can be characterized by a variety of pharmacological properties. The phytocompound has proven to have antiinflammatory and antioxidant properties, antibacterial, antiviral, antifungal, and antiparasitic activities [19]. It has been reported to decrease the level of LDL cholesterol, total cholesterol, and triglyceride and directly correlated to inhibit the process of atherosclerosis [20]. It has been showed that API has protective properties in myocardial injury [21] and is also effective in neuronal ischemia [19]. Salehi et al. have presented in their research paper that API improves renal disfunction and lowers the blood glucose in diabetes, attenuates the stress and depression symptoms, and has applicability in diseases like Alzheimer or amnesia by increasing the capacity of learning and memory skills [22] The authors also presented various clinical trials that moni- tored the beneficial effects of API supplementation in different pathologies (Alzheimer's disease, anxiety, depression, insomnia, and knee osteoarthritis) [22]. Zhou et al. have described that the phytocompound protects the endocrine system and exerts a positive role in osteoporosis by inhibiting the osteoclast and activation of osteoblasts. Also, one of the most studied properties is cytostatic and cytotoxic effects in different types of cancer [23].

The in vitro antitumoral effect of API has been studied and confirmed by many researchers on different cancer cell lines. Madunić et al. revealed that API (10 to $100 \mu \mathrm{M})$ can induce morphological changes in MDA-MB-231 human breast cancer cell line in a time- and dose-dependent manner. Moreover, using the Annexin V/FITC assay, they have shown that API $(20,40$, and $100 \mu \mathrm{M})$ promotes the induction of late apoptotic death in MCF-7 cells. On the other hand, only the highest tested concentrations $(60$ and $100 \mu \mathrm{M})$ triggered the same mode of death in MDA-MB-231 cells [24]. Seo et al. demonstrated that the phytocompound at different concentrations $(10,20,40$, and $80 \mu \mathrm{M})$ influenced the inhibition of human prostate cancer cell line (PC3 and LNCap) proliferation after induction of apoptosis which was possible through increased p21 pathway and decreased PLK-1 expression as a result of apoptotic cell death [25]. In their study, Bai et al. proved that API could selectively inhibit different tumor cells. In the screened interval of concentrations $(20-100 \mu \mathrm{M})$, the phytocompound induced apoptosis of MCF-7 breast cancer cells, while the same concentration caused only a little damage on MCF-10A normal cells. The authors also indicated that API $(80 \mu \mathrm{M})$ induces early apoptotic events (from $1.1 \%$ to $18.6 \%)$. Moreover, the highest tested concentration $(100 \mu \mathrm{M})$ after an incubation period of $24 \mathrm{~h}$ induced a significant increase in late apoptosis and necrosis for MCF-7 cells (from 1.6\% to 17.2\%) [26]. Lindenmeyer et al. showed that treatment with this natural phytocompound $(22.8-45.5 \mu \mathrm{M})$ strongly inhibits the human MDA-MB-231 breast cancer cell invasion, migration, and also proliferation, events correlated to cell cycle arrest in the G2/M phase [27]. Since API has generated a great deal of interest, in a recent study, Liu et al. have demonstrated that this compound inhibits the proliferation of HeLa cervical cancer cell line with an $\mathrm{IC}_{50}=47.26 \mu \mathrm{M}$. The mechanism is based on inducing apoptosis and cell cycle arrest at G0/G1 phase. The authors reported that the cell proliferation is mainly related to the signalling pathways PI3K/AKT (intracellular signal transduction pathway) and PTEN (natural inhibitor). In this case, the PTEN/PI3K/AKT pathway was inhibited in a concentration-dependent manner, and cell migration was also impeded via matrix metalloproteinases 2 and 9 [28]. Up to date, Chen et al. demonstrated the anticancer activity of API using human HT-29 colon cancer cell line in vitro and also in vivo in xenografted mice models. Their results indicate that API at 20, 40 , and $80 \mu \mathrm{M}$ in vitro and $35 \mathrm{mg} / \mathrm{kg}$ in vivo inhibits the proliferation of cancer cells and the growth of xenografted tumors. The same study reports the inhibition of some signalling pathways which play an essential role in the proliferation of cancer cells, namely, p-mTOR, p-PI3K, and p-AKT expressions in HT-29 cells concentrationdependently [29]. 
Nowadays, cancer is a pathology that affects people worldwide and is one of the main cause of death. One of the most aggressive types of cancer is represented by melanoma. The particularity of this type of skin cancer is given by a big metastatic capacity. The development of melanoma is linked also to the UV exposure because of the damages occurred on the DNA. One of the most representative classes of natural compounds with ability to absorb UV radiation is the flavonoids [30].

Researchers like Xu et al. showed that API has the capacity to affect cell proliferation (API $60 \mu \mathrm{M}-24 \mathrm{~h}$ ) and also has proapoptotic properties (API $30 \mu \mathrm{M}-24 \mathrm{~h}$ ) on melanoma cell lines (A375, A2058, and RPMI-7951) [31]. Other groups have proven that API inhibited cell proliferation (API 40$160 \mu \mathrm{M}-48 \mathrm{~h})$ on A375 and C8161 melanoma cell lines, affected the capacity of cell migration and invasion $(40 \mu \mathrm{M}$ $72 \mathrm{~h}$ ), and had proapoptotic effect ( 40 and $100 \mu \mathrm{M}-24 \mathrm{~h}$ ) when tested in vitro [32]. Das et al. demonstrated that API inhibited cell proliferation $(20-250 \mu \mathrm{g} / \mathrm{mL}-24 \mathrm{~h})$ and increased the number of cells in early and late apoptosis by damaging the normal function of the mitochondria $(20,50$, and 80 $\mu \mathrm{g} / \mathrm{mL}-24 \mathrm{~h}$ ) [33]. Chan et al. have noticed the antitumoral effect of API (10 and $20 \mu \mathrm{M})$ on A431 squamous cell carcinoma by inducing cell cycle arrest in phase G2/M [34].

Tumor formation, development, progression, and metastasis require an activated angiogenesis process. Thus, targeting angiogenesis is included in the current anticancer therapeutic strategies. Natural compounds are being intensively researched as safer alternatives with multiple targeting potential. API was reported as an angiogenesis inhibitor in several types of cancers [35]. Still, the in vivo efficacy of API as antiangiogenic in melanoma was less investigated.

Regarding the fact that melanoma is one of the most aggressive type of skin cancer, many studies approached this topic. The researchers have proven the antiproliferative effect of API (10-50 $\mu \mathrm{M}-24 \mathrm{~h}, 48 \mathrm{~h}$, in a dose-dependent manner) on B16F10 murine cell melanoma line [36]. The antiproliferative and cytotoxic effect of API have been demonstrated also by testing this phytocompound ( 30 and $60 \mu \mathrm{M}-72 \mathrm{~h}$ ) on B164A5 murine melanoma cell line. The obtained results were in a dose-dependent manner [37]. Cao et al. have showed that API $(150 \mathrm{mg} / \mathrm{kg})$ has the ability to inhibit metastasis on B16F10 murine melanoma cell line. Moreover, in case of human melanoma cell lines A375, 518A2, and G361, API $(40 \mu \mathrm{M})$ inhibited in a dose-dependent manner the expression levels of STAT3 [38].

Malignant melanoma is one of the most aggressive forms among all types of cancer, and while significant advances in therapeutic management have improved melanoma patient care, there are still major clinical challenges such as drug resistance and recurrence to be overcome [39] API was investigated here, considering the promising anticancer activity by targeting several signalling pathways, next to limited secondary effects that were previously reported [32, 37, 38]. In previous studies, we established that API elicited in a dose-dependent way antimigratory and proapoptotic effects on different melanoma cells, on A375 human melanoma cell line [37], and on B164A5 murine melanoma cells [40].
Given the high impact of nanotechnologies, future perspectives could involve the evaluation of nanoformulations that contain API, a direction that could improve the biocompatibility and enlarge the spectrum of biological activities [41]. Some formulations that contain API already showed an improvement in solubility, bioavailability, and antioxidant properties [42]. These include nanosuspensions of API, nanocrystals of API, and API-loaded carbon nanotubes. API-loaded nanoparticles proved to reduce inflammation and fibrosis in vitro in chronic pancreatitis, and bovine serum albumin nanoparticles with API had a beneficial effect in lung injury. Nanoencapsulation of API in different nanoparticles improved the antitumor effect of API in various types of cancers [42]. Further in vivo and clinical studies are needed in order to establish the potential of nanoformulations that contain API in different pathologies.

Although the antiproliferative and antiangiogenic effects of API were studied on a significant number of human and murine melanoma cell lines, the intention of this study was to enrich the existing knowledge on the melanoma potential of API by exploring the impact of the compound on SKMEL-24 human metastatic melanoma cell line, investigating in vitro, the cytotoxicity, viability, and antimigration effects, and in vivo on the chick chorioallantoic membrane, the antiangiogenic, and anti-invasion potential. Given the aggressive form of melanoma, we chose a human melanoma cell line derived from metastatic site, the lymph node.

\section{Materials and Methods}

2.1. Cell Culture. The cell line SK-MEL-24 human melanoma (ATCC ${ }^{\circledR}$ HTB-71) was acquired from the American Type Culture Collection (ATCC). Cells were cultured in Eagle's Minimum Essential Medium (EMEM; ATCC) supplemented with $15 \%$ fetal bovine serum (FBS; Sigma-Aldrich, Germany) and $1 \%$ penicillin/streptomycin mixture (Pen/Strep, 10,000 IU/mL; Sigma-Aldrich, Germany). Cells were maintained under standard conditions: $37^{\circ} \mathrm{C}$ and a humidified atmosphere of $5 \% \mathrm{CO}_{2}$ in a Steri-Cycle i160 incubator (Thermo Fisher Scientific, USA).

2.2. MTT Assay. The effect of API (purchased from SigmaAldrich) on SK-MEL-24 human melanoma cells viability was evaluated by using the (3-(4,5-dimethylthiazol-2-yl)2,5-diphenyltetrazolium bromide (MTT) assay. The method was performed as previously described [37]. In brief, a number of $1 \times 10^{4}$ cells/well were seeded onto 96-well culture plates and allowed to adhere overnight. The next day, the cells were stimulated with different concentrations of API $(0.3,1,3,10,30$, and $60 \mu \mathrm{M})$ and further incubated for $72 \mathrm{~h}$. After $72 \mathrm{~h}$ of incubation, a volume of $10 \mu \mathrm{L}$ of $5 \mathrm{mg} / \mathrm{mL}$ MTT solution from the MTT kit (Sigma-Aldrich) was added in each well and incubated for an additional $3 \mathrm{~h}$. The formed formazan crystals were dissolved by adding $100 \mu \mathrm{L} /$ well of lysis solution provided in the MTT kit. The cells treated with the solvent dimethyl sulfoxide (DMSO, Sigma-Aldrich) were used as the control group. The absorbance was spectrophotometrically analyzed at $570 \mathrm{~nm}$ with a microplate reader (BioRad, xMark Microplate Spectrophotometer). 
2.3. Evaluation of the Cytotoxic Potential by Lactate Dehydrogenase (LDH) Assay. The cytotoxic rate of API at the highest tested concentrations ( 30 and $60 \mu \mathrm{M})$ was evaluated by the quantification of the $\mathrm{LDH}$ amount released into the medium following cellular membrane damage. In order to perform the LDH assay (Cytotoxicity detection kit, 11644793001, Roche), a number of $5 \times 10^{3}$ cells/well were seeded onto 96-well culture plates and allowed to adhere overnight. The next day, the cells were stimulated with the highest tested concentrations of API ( 30 and $60 \mu \mathrm{M})$ and further incubated for $72 \mathrm{~h}$. After the incubation period, a volume of $100 \mu \mathrm{L}$ was transferred from each well into a 96-well culture plate and mixed with $100 \mu \mathrm{L}$ of reaction mixture (obtained according to the manufacturer's instructions) and incubated for $30 \mathrm{~min}$ at room temperature.

The amount of LDH leakage in the medium was determined by measuring the absorbance at two wavelengths $(490 \mathrm{~nm}$ and $680 \mathrm{~nm})$ using a microplate reader (BioRad, xMark Microplate Spectrophotometer). In order to determine the spontaneous and maximum release of $\mathrm{LDH}$, untreated cells (low control) and cells treated with $1 \%(v / v)$ Triton- X100 (high control) were used.

2.4. Scratch Assay. In order to assess SK-MEL-24 cell invasion capacity after stimulation with the highest tested API concentrations (30 and $60 \mu \mathrm{M}$ ), a scratch assay was performed. Briefly, a number of $2 \times 10^{5}$ cells/well were plated onto 12-well culture plates until a confluent cell monolayer was reached. Afterwards, a sterile pipette tip was used in order to draw a gap in the middle of each well. All the cells and cellular debris that detached following the procedure were gently washed with phosphate buffer saline (PBS). Further, the cells were stimulated with API $(30$ and $60 \mu \mathrm{M})$. Pictures of the scratch area were taken at $0 \mathrm{~h}$ and $24 \mathrm{~h}$ at a magnification of $10 x$ by means of an inverted microscope (Olympus IX73) equipped with DP74 camera (Olympus, Tokyo, Japan). For analyzing the cell growth, the cellSense Dimension software was used.

The following formula was used in order to calculate the scratch closure rate [43]:

$$
\text { Scratch closure rate }=\left[\frac{A_{t 0}-A_{t}}{A_{t}}\right] * 100,
$$

where $A_{t 0}$ is the scratch area at time $0 \mathrm{~h}$ and $A_{t}$ is the scratch area at $24 \mathrm{~h}$.

2.5. Chorioallantoic Membrane Assay (CAM). The CAM assay was used to investigate the effect of API on the angiogenic process under tumor development, using SK-MEL-24 human melanoma cells. This in vivo protocol is widely used to study neovascularisation in a tumor microenvironment and the potential inhibitors of new vessel formation. Using a slightly modified technique developed by Ribatti et al. [44], fertilized hen (Gallus gallus domesticus) eggs were disinfected and incubated at $37^{\circ} \mathrm{C}$ and $50 \%$ humidity. The protocol includes the removal of $3-4 \mathrm{~mL}$ of albumen on the third day of incubation, followed by cutting a window on the upper side of the eggs on the following day.
SK-MEL-24 melanoma cells cultured according to the above-described protocol were inoculated on top of the developing CAM on day 10 of incubation (day $0,0 \mathrm{~h}$ ) [45]. After detaching the cells from the culture plate by trypsinization, they were cleansed and resuspended in the culture medium until reaching the final concentration of $10^{4} / 5 \mu \mathrm{L}$. A volume of $5 \mu \mathrm{L}$ of the SK-MEL-24 cell suspension was inoculated inside a plastic ring previously placed on each CAM. API in concentration of $30 \mu \mathrm{M}$ and $60 \mu \mathrm{M}$, next to DMSO $0.5 \%$ as solvent control, was administered daily inside the melanoma cells-containing plastic rings. All specimens were monitored in ovo, and representative images were registered by means of a stereomicroscope (Discovery 8 Stereomicroscope, Zeiss) connected to digital camera Axiocam 105 color; software AxioVision SE64. Rel. 4.9.1 Software (Göttingen, Germany) and the images were processed by the Zeiss ZEN software, ImageJ (Image J Version 1.50e https://imagej .nih.gov/ij/index.html), and GIMP (GIMP v 2.8 GNU Image Manipulation Program https://www.gimp.org/).

2.6. Statistical Analysis. The data obtained in the in vitro section was analyzed using GraphPad Prism 5.0 (GraphPad Software, CA, USA). Comparison among groups was performed by means of One-way ANOVA test followed by Dunnett's multiple comparison posttest $\left({ }^{* * *} p<0.001\right.$ vs. control).

\section{Results}

3.1. MTT Assay. The MTT assay illustrates the cell viability percentage of the human melanoma SK-MEL-24 cells. The effect of API was assessed on SK-MEL-24 cells after a $72 \mathrm{~h}$ stimulation period and compared to control. Figure 1 depicts the effect of API $(0.3,1,3,10,30$, and $60 \mu \mathrm{M})$ on melanoma cell viability. Obtained results indicate that in the range of tested concentrations, there is a decrease in tumor cell viability with the most significant reduction noticed at the two highest used concentrations (for $30 \mu \mathrm{M}$, tumor cell viability was $75.08 \pm 5.5 \%$ vs. control, and for $60 \mu \mathrm{M}$, tumor cell viability was $62.9 \pm 5.4 \%$ vs. control).

3.2. Assessment of the Cytotoxic Potential by means of $L D H$ Release. Together with proliferation, cell cytotoxicity assays are frequently employed for drug screening, including natural molecules in order to detect whether the test compounds have effects. The cytotoxic potential of API determined by $\mathrm{LDH}$ assay is represented in Figure 2. The two highest concentrations of API ( 30 and $60 \mu \mathrm{M}$ ) were selected due to the fact that the percent of viable cells observed through MTT assay was significantly lower following the stimulation. As shown in the graphic, a significant dose-dependent cytotoxic effect was provoked by API. At $30 \mu \mathrm{M}$, the cytotoxic rate was $9 \pm 1.1 \%$ vs. control $(1.6 \pm 0.7 \%)$, whereas at the highest dose tested, $60 \mu \mathrm{M}$ the cytotoxic rate was slightly higher, $11.1 \pm$ $2.4 \%$ vs. control.

3.3. Scratch Assay. It has been already demonstrated that migration of cancer cells is a prerequisite for tumor cell invasion and metastasis, a totally unwanted event, antimigratory potential being a desirable property for an anticancer agent. 


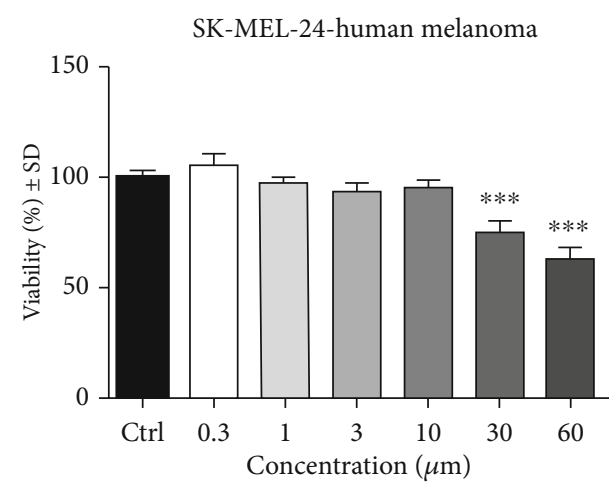

Figure 1: SK-MEL-24 human melanoma cell viability after $72 \mathrm{~h}$ stimulation with API $(0.3,1,3,10,30$, and $60 \mu \mathrm{M})$. Results are expressed as cell viability percentage (\%) related to control cells. Comparison among groups was performed by means of One-way ANOVA test followed by Dunnett's multiple comparison posttest $\left({ }^{* * *} p<0.001\right.$ vs. control).

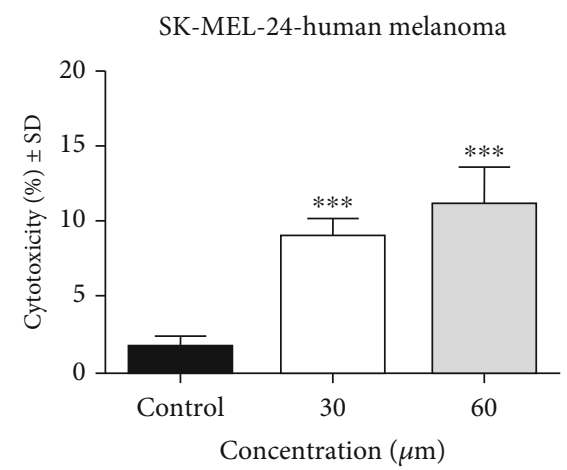

FIgURE 2: The cytotoxic effect of API (30 and $60 \mu \mathrm{M})$ on SK-MEL24 human melanoma cells after a stimulation period of $72 \mathrm{~h}$. The results are expressed as cytotoxicity percentage (\%) related to the control cells. Comparison among groups was performed by means of One-way ANOVA test followed by Dunnett's multiple comparison posttest $\left({ }^{* * *} p<0.001\right.$ vs. control).

The antimigratory potential of API at the highest tested concentrations (30 and $60 \mu \mathrm{M}$ ) on SK-MEL-24 melanoma cells was determined by means of scratch assay. Figure 3 shows that API elicited an inhibition of tumor cells migration in a dose-dependent manner. Figure 3(a) depicts the effect of API at $0 \mathrm{~h}$ and $24 \mathrm{~h}$, respectively, poststimulation. At the highest concentration tested, a slight change in cell shape can be noticed-some of them showed round shape and started to detached. Figure 3(b) presents the scratch closure rate for the two concentrations used compared to control. At $30 \mu \mathrm{M}$, API produced a scratch closure rate of only $6.2 \%$ and for $60 \mu \mathrm{M}$ an even lower one, namely, 3.4\%. This effect indicates that API reduced melanoma cell migration.

\subsection{API Effects on SK-MEL-24 Angiogenesis Using the CAM} Assay. It is very well known that an activated angiogenesis process is required for tumor formation, development, progression, and metastasis. To assess the potential antiangiogenic effect of API in a melanoma microenvironment, we used the tumor CAM assay, with SK-MEL-24 human mela- noma cells. Observing the control group, where only $0.5 \%$ DMSO was applied, the development of tumor cells was not affected, and cell area extended progressively. After 24 hours of incubation with the compound, only some compact tumor cell areas were noticed inside the application ring, while after 48 hours postinoculation, a large and compact tumor area covered most of the inner surface of the ring, and extended areas of tumor cells were present outside the ring. Blood vessels formed a densely network in a spokes wheel pattern towards the application ring (Figure 4).

API in both tested concentrations limited melanoma cell growth and migration and induced a reduced angiogenic reaction. As shown in Figure 4, after incubation with API for $48 \mathrm{~h}$, tumor cell areas were limited, and only a few scattered cells were observed outside the application spot. The number of capillaries was decreased compared to the control sample; the vessels in peritumoral areas had a narrow aspect and showed a low branching profile. Minimal differences could be observed between the two tested concentrations of API, with the higher concentration inducing a slightly higher decrease in tumor cell development and attenuation of neovascularisation.

\section{Discussion}

Nowadays, a large number of studies including in vitro, in vivo, and clinical trials highlight the fact that API is a potential therapeutic agent used for the management of several diseases [46].

Regarding the anticancer activities, in recent years, API has gained a significant importance as a health promoting agent due to its reduced toxicity and due to the fact that this phytocompound does not affect healthy cells but is active on various cancer cell lines [47].

The major findings of the present study indicate that in the screened conditions API reduced SK-MEL-24 melanoma cell migration and viability in a dose-dependent manner, the most significant results being obtained at 30 and $60 \mu \mathrm{M}$. Furthermore, a cytotoxic effect was obtained at 30 and $60 \mu \mathrm{M}$ determined by LDH release. To the best of our knowledge, this is the first study that presents the effects of API on SKMEL-24 human metastatic melanoma cells. Our data are in accordance with the scientific literature where it is indicated that this flavone has an antiproliferative effect against different melanoma cell lines [32, 37, 40]. Our research group has previously established that API $(0.3-60 \mu \mathrm{M})$ is a potential antiproliferative and proapoptotic agent against A375 human melanoma cell line, with an $\mathrm{IC}_{50}$ of $33.02 \mu \mathrm{M}$ [37]. Following the same thought, Woo et al. investigated the effect of API $(25,50,75$, and $100 \mu \mathrm{M})$ for $24 \mathrm{~h}$ on human melanoma cells (A375P and A375SM). Using in vitro assays, this research group has shown that the cell viability was decreased, and the expression of Bax, p53, PARP, and cleaved caspase- 9 increased in a dose-dependent manner [48]. Using A2058 and A375 melanoma cell lines, Hasnat et al. pointed out that API treatment $(10,20$, and $50 \mu \mathrm{M})$ inhibited the growth of melanoma cells. Moreover, API treatment increased apoptotic factors such as caspase-3, cleaved poly (ADP-ribose) polymerase, and also modulation of FAK/ERK 
Control

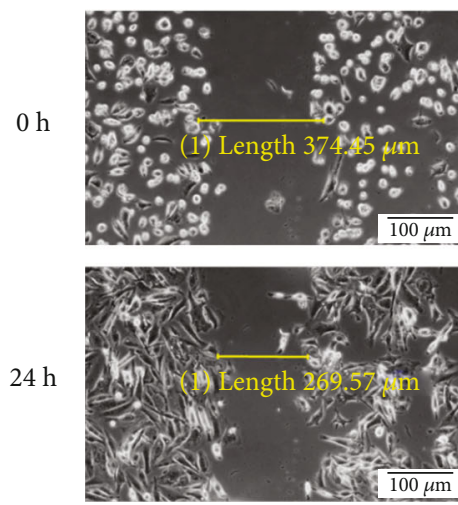

API $30 \mu \mathrm{m}$
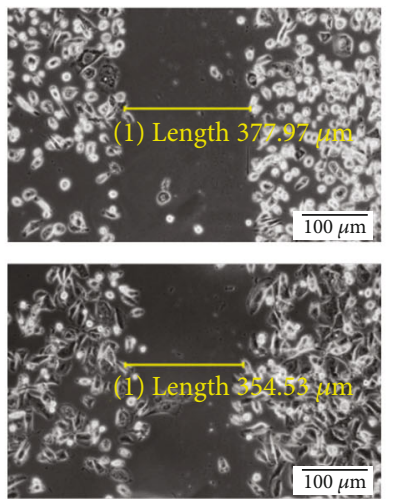

(a)
API $60 \mu \mathrm{m}$
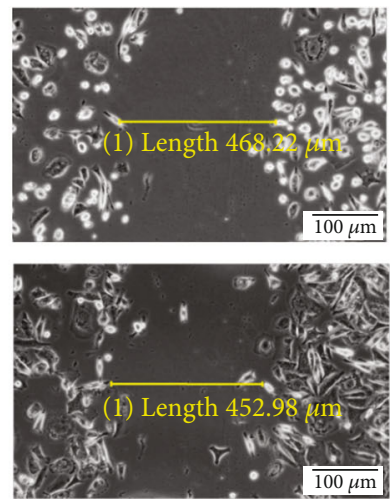

SK-MEL-24 cell line

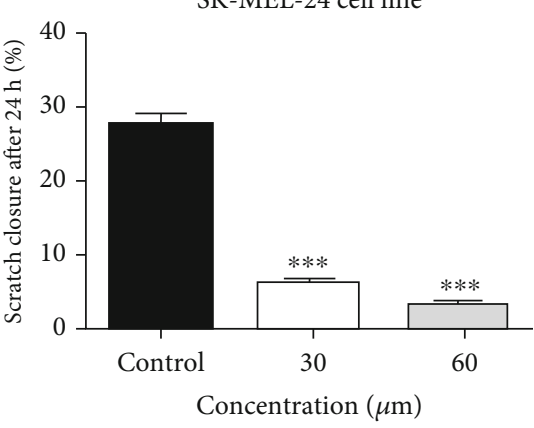

(b)

Figure 3: (a) The effect of API (30 and $60 \mu \mathrm{M})$ on SK-MEL-24 human melanoma cell migration capacity. Images were taken by light microscopy at 10x magnification. Melanoma cell migration was monitored by imaging the scratch line initially and at $24 \mathrm{~h}$ poststimulation. (b) The bar graphs are expressed as percentage of scratch closure after $24 \mathrm{~h}$ compared to the initial surface ( $0 \mathrm{~h}$ ). One-way ANOVA test and Dunnett's multiple comparison posttest were used for comparison among groups $\left({ }^{* * *} p<0.001 \mathrm{vs}\right.$. control).

and integrin signalling pathways [49]. The phytocompound also exhibited proapoptotic properties on A375, A2058, and RPMI-7951 melanoma cells. The researchers showed that API $(30 \mu \mathrm{M})$ presented inhibitory effects on the expression levels of PD-L1 and stimulation of IFN- $\gamma$ [31]. Another study investigated the effects of this natural molecule on B16F10 melanoma cells. The research group showed that API in the dose ranges of 10-50 $\mu \mathrm{M}$, after incubation periods of 24 and $48 \mathrm{~h}$, presented significant increase of cell proportion in the G0/G1, S, and G2/M phases followed by a significant decrease in the G0/G1 phase [36].

Deepening the experimental line, Woo et al. transplanted the A375SM human melanoma cells in nude mice which were treated intraperitoneally with API (25 and $50 \mathrm{mg} / \mathrm{kg}$ ). Results showed that the tumor size and weight were significantly decreased in a dose-dependent manner in the API treated groups when compared with the control group [48]. $\mathrm{Cao}$ et al. demonstrated the antimetastatic effect of API in vivo using C57BL/6 mice. Results showed that in the murine melanoma B16F10 cell line, API (150 mg/kg) presented antimetastatic activity by suppressing STAT3 phosphorylation and downregulating MMP-2, MMP-9, and VEGF pathways [38]. Xu et al. investigated API effects in vivo on melanoma xenograft mouse models using the B16-F10 melanoma cell line. This group suggested that API treatment $(150 \mathrm{mg} / \mathrm{kg})$ significantly suppressed the growth of B16-F10 xenograft tumors and regulated the sensitivity of tumor to immune killing by increased the abundance of CD4+ and CD8+ T cells [31].

Various research paper showed the in vitro antitumor effect of API on several types of cancer cells, including MDA-MB-231 and MCF-7 human breast cancer cell lines [24, 26, 27], PC3 and LNCap human prostate cancer [25], HeLa cervical cancer cell line [28], and HT-29 colon cancer cell line [29].

Other in vivo studies also pointed out that intraperitoneally administered API can attenuate the tumor growth in B16-BL6 murine melanoma metastasis model (50, 25, and $12.5 \mathrm{mg} / \mathrm{kg}$ ) [50], Lewis lung carcinoma (LLC) and DHDK12 colonic cancer line $(50 \mathrm{mg} / \mathrm{kg} /$ day $)$ [51], pancreatic cancer PCS (50 mg, once daily) [52], human hepatocellular carcinoma cells (HepG2-fLuc and HepG2-GFP cells) $(0.5 \mathrm{mg} / \mathrm{kg} / 2$ days) [53], renal cell carcinoma (RCC) cells (30 mg/kg every 3 days for 21 days) [54], colorectal adenocarcinoma DLD1 and SW480 cells $(20 \mathrm{mg} / \mathrm{kg}$ once a week) [55], human ovarian cancer A2780 cells ( $5 \mathrm{mg} / \mathrm{kg}$ body weight) [56], human MDA-MB-231 breast cancer cells (5 or $25 \mathrm{mg} \cdot \mathrm{kg}^{-1}$ every day for 8 weeks) [57], and colorectal cancer $(20 \mathrm{mg} / \mathrm{kg}$ ) [58].

ROS (reactive oxygen species) have been reported as an important regulator for mitochondrial function [59]. 

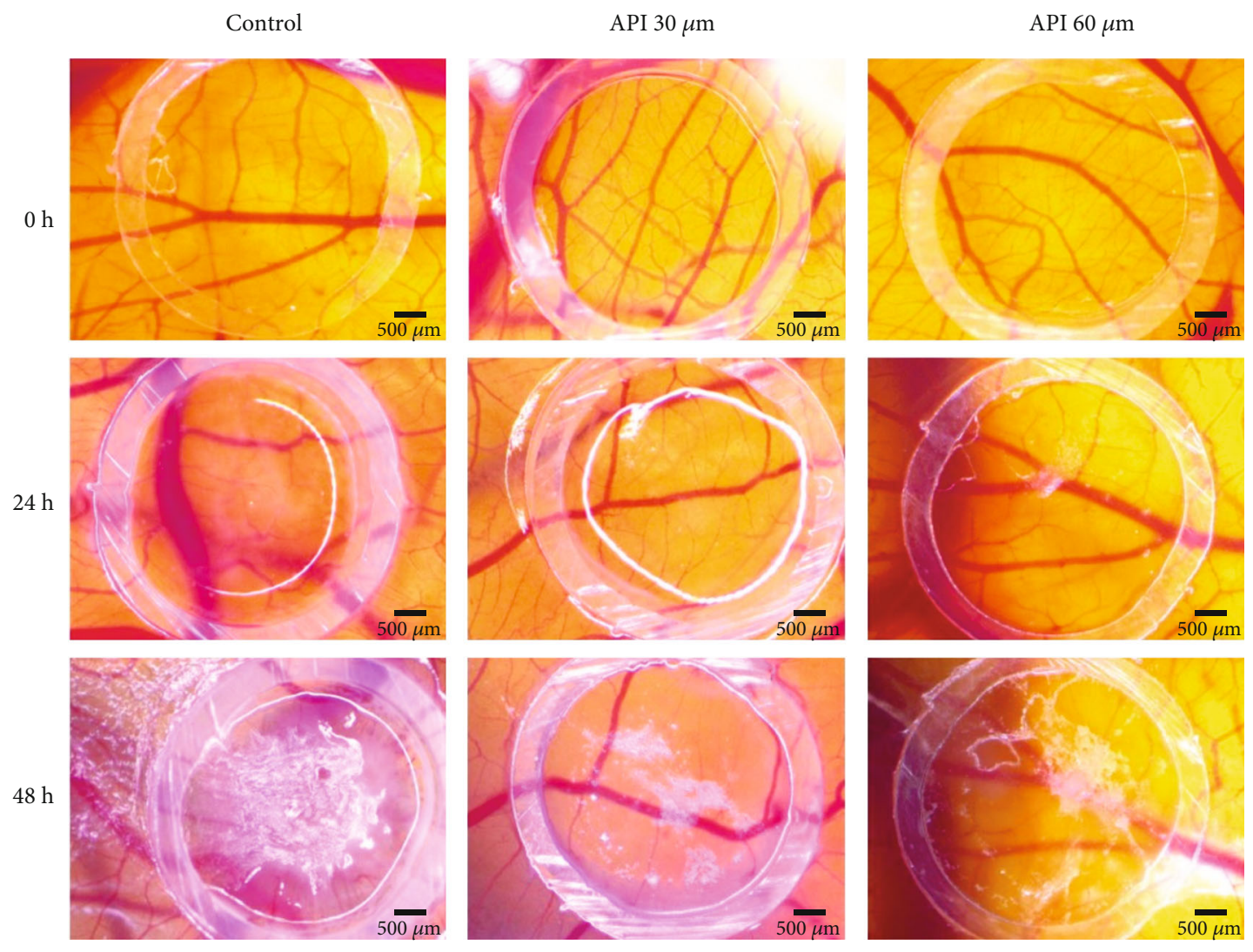

FIgURE 4: The antiangiogenic effect of API (30 and $60 \mu \mathrm{M})$ using a SK-MEL-24 tumor CAM assay. Representative stereomicroscope images were registered initially $(0 \mathrm{~h})$, after $24 \mathrm{~h}$, and $48 \mathrm{~h}$ after inoculation. Scale bars represent $500 \mu \mathrm{m}$.

Furthermore, the overproduction of ROS can damage cellular components such as proteins, lipids, and DNA and also plays a significant role in apoptosis induction or can induce cell death. Along the same line of thought, Bai et al. showed that API treatment increases the ROS level which led to apoptosis in the API-treated MCF-7 cells [26].

In different cancer cells, high levels of ROS (reactive oxygen species) can appear from increased metabolic activity and mitochondrial function and also from increased activity of oxidases, cyclooxygenases, lipoxygenases, and cellular receptor signalling or through oncogene activity. Furthermore, the overproduction of ROS can damage cellular components such as proteins, lipids, and DNA and also plays a significant role in apoptosis induction or can induce cell death $[60,61]$. Along the same line of thought, Bai et al. showed that API treatment increases the ROS level which leads to apoptosis in the API-treated MCF-7 cells [26].

Tumor necrosis factor- (TNF-) related apoptosisinducing ligand (TRAIL) is an encouraging anticancer agent which induces apoptotic cell death in numerous cell lines by creating a complex with death receptors. This complex is able to trigger the activation of the death-inducing signalling complex and also the caspase signalling cascade [62]. Taken together these findings, researchers reported that API upregulates ROS generation; however, the ROS inhibitors (glutathione and $\mathrm{N}$-acetyl-L-cysteine) present a moderate increase of API/TRAIL-induced apoptosis by a slight increase of ROS generation. The results were compared with rapamycin (autophagy inducer) which improved apoptosis by a slight increase of ROS generation [63]. The same conclusion was drawn by Shukla et al. In their approach, the research group indicates that API $(10-80 \mu \mathrm{M})$ induces apoptosis in human 22Rv1 prostate cancer cells. They have shown that this event is initiated by ROS-dependent disruption of the mitochondrial membrane potential by transcriptional-dependent and -independent p53 pathways in a dose- and time-dependent manner. Moreover, API treatment increased the level of cleaved caspase- 3 and modified Bax and Bcl-2 protein levels, whereon favor apoptosis [64].

There is clear evidence that API exert antiproliferative and proapoptotic effects by multiple and complex mechanism. The key signalling pathways by which API employ its anticancer properties are highlighted mainly by the upregulated levels of caspase [65], Bax, and PARP levels [66]; therefore decrease Bcl-2 and Mcl-1 levels [33, 44, 67] decreased the cytochrome $\mathrm{C}$ in the mitochondrial fraction [67]; inhibited NF- $\kappa \mathrm{B}$ activation [68]; suppressed the expression of cyclin B1, Cdc2, and Cdc25c; induced PARP cleavage; and induced LC3-II [69].

In order to gain more details regarding the effect of API on the metastatic human melanoma cell line, we performed the tumor chorioallantoic membrane assay. Considered as valuable intermediate in vivo biological tool as a preliminary assay to rodent in vivo animal assays, the model has several advantages, including costs, time, simplicity, and reproducibility [45] API was investigated as an antiangiogenic agent 
with potential benefits in hindering cancer progression and metastasis for several types of cancer by targeting several signalling pathways [70]. API has proven to be involved in the inhibition of angiogenesis by suppressing expression of VEGF and HIF- $1 \alpha$ [71], inactivation of ERK1/2 [72], inhibition of STAT3 signalling, and decreasing the levels of the MMP-2 and MMP-9 expression [38]. Less data is available regarding the effects of API on the angiogenesis process associated to human melanoma microenvironment. Our previous investigation on API effects on an A375 melanoma model showed the potential effect of decreasing angiogenic and tumor progression [37]. The results obtained here on an SK-MEL-24 melanoma model using the chorioallantoic membrane assay come to emphasise that treatment with 30 and $60 \mu \mathrm{M}$ API induces a reduced neovascularisation next to limiting melanoma cell growth and migration.

API at 30 and $60 \mu \mathrm{M}$ reduced SK-MEL-24 melanoma cell viability and migration and produced slight changes in melanoma cell morphology at $24 \mathrm{~h}$ poststimulation. In addition, in the tumor chorioallantoic membrane, API decreased tumor cell development. Once more, API showed an antitumor effect on the melanoma cells. In the upcoming studies, we will address the effect of API in vivo, using experimental animal models. Furthermore, for a targeted drug delivery in melanoma, various nanoformulations are taken into consideration.

\section{Conclusion}

Results from the present study show that API in the tested dose ranges presents in vitro antiproliferative properties against SK-MEL-24 human metastatic melanoma cell line. At 30 and $60 \mu \mathrm{M}$, API elicited a dose-dependent cytotoxic effect and showed antimigratory potential. Moreover, treatment with 30 and $60 \mu \mathrm{M}$ API using the tumor chorioallantoic membrane assay induces a reduced neovascularisation next to limiting melanoma cell development; hence, API could be considered as a promising antiangiogenic agent in malignant melanoma. Overall, API proved to possess antitumor effect on SK-MEL-24 melanoma cells, an effect that can be assessed in future studies. Moreover, due to the impact of nanotechnologies, future directions could involve the design of nanoformulations such as apigenin-conjugated silver or gold nanoparticles in order to increase the bioavailability of this flavone for in vivo experimental models and also to expand the spectrum of biological activities [41]. Thus, API can become a valuable tool in the management of chronic diseases such as cardiovascular diseases, diabetes, and neurological diseases. As indicated in various studies [41, 42], the use of natural compounds or of various nanoformulations that include them could be a beneficial strategy, benefitting from higher biocompatibility, biodegradability, and biomimetic features. Further clinical trials involving API could be performed as a possible strategy for the management of melanoma. Since the number of articles involving the study of nanotechnology in humans is lower compared to nonnanotherapies, an evaluation of API in such a manner could be of real interest.

\section{Abbreviations}

$\begin{array}{ll}\text { BAX: } & \text { Bcl-2-associated X protein } \\ \text { Bcl-2: } & \text { B-cell lymphoma 2 } \\ \text { Cdc2 and Cdc25c: } & \text { Cell division cycle } \\ \text { P53: } & \text { Cellular tumor antigen } \\ \text { PARP: } & \text { Poly-ADP ribose polymerase } \\ \text { FAK/ERK: } & \text { Adhesion-mediated focal adhesion } \\ & \text { kinase } \\ \text { IFN- } \gamma: & \text { Interferon gamma } \\ \text { STAT3: } & \text { Signal transducer and activator of tran- } \\ & \text { scription 3 } \\ \text { MMP-2, MMP-9: } & \text { Matrix metallopeptidase } \\ \text { VEGF: } & \text { Vascular endothelial growth factor } \\ \text { mTOR: } & \text { Mammalian target of rapamycin } \\ \text { NF- } \kappa \text { B: } & \text { Nuclear factor kappa } \\ \text { p-PI3K: } & \text { Phosphatidylinositol 3-kinase } \\ \text { p-AKT: } & \text { Protein kinase B } \\ \text { LC3-II: } & \text { Cytosolic form of LC3 } \\ \text { TNF: } & \text { Tumor necrosis factor } \\ \text { TRAIL: } & \text { Tumor necrosis factor- (TNF-) related } \\ & \text { apoptosis-inducing ligand. }\end{array}$

\section{Data Availability}

All data used to support the findings of this study are included within the article.

\section{Conflicts of Interest}

The authors declare that there is no conflict of interests regarding the publication of this paper.

\section{Acknowledgments}

This research was founded by the "Victor Babeş" University of Medicine and Pharmacy grant number 3POSTDOC/1238/2020 offered to Assoc. Prof. Corina Danciu.

\section{References}

[1] K. Suresh, P. Surender, K. W. Yogesh, K. Aditya, and S. Parul, "Therapeutic potential of medicinal plants: a review," Journal of Biological and Chemical Chronicles., vol. 1, no. 1, pp. 4654, 2015.

[2] World Health Organization, WHO global report on traditional and complementary medicine, Geneva, 2019.

[3] P. C. Chikezie and O. A. Ojiako, "Herbal medicine: yesterday, today and tomorrow," Alternative and Integrative Medicine, vol. 4, no. 3, 2015.

[4] P. Dinesh and M. Rasool, "Herbal formulations and their bioactive components as dietary supplements for treating rheumatoid arthritis," in Bioactive Food Dietary Intervention for Arthritis and Relateted Inflammatory Diseases, pp. 385-399, Academic Press, 2019.

[5] J. Madunic, I. V. Madunic, G. Gajski, J. Popic, and V. GarajVrhovac, "Apigenin: a dietary flavonoid with diverse anticancer properties," Cancer Letters, vol. 413, pp. 11-22, 2018.

[6] X. Yan, M. Qi, P. Li, Y. Zhan, and H. Shao, "Apigenin in cancer therapy: anti-cancer effects and mechanisms of action," Cell \& Bioscience, vol. 7, no. 1, 2017. 
[7] T. A. Stump, B. N. Santee, L. P. Williams et al., "The antiproliferative and apoptotic effects of apigenin on glioblastoma cells," Journal of Pharmacy and Pharmacology, vol. 69, no. 7, pp. 907-916, 2017.

[8] C. Kanadaswami, L. Lee, P. H. Lee et al., "The antitumor activities of flavonoids," In Vivo, vol. 19, pp. 895-910, 2005.

[9] P. Batra and A. K. Sharma, "Anti-cancer potential of flavonoids: recent trends and future perspectives," 3 Biotech, vol. 3, no. 6, pp. 439-459, 2013.

[10] Cancer trials- Genistein [Internet]https://clinicaltrials.gov/ct2/ show/NCT00584532?term=genistein \& cond=Cancer + Prostate\&draw $=2 \&$ rank $=2$.

[11] Clinical trials- Desmodium-Breast cancer [Internet]https:// clinicaltrials.gov/ct2/show/study/NCT03959618?term= desmodium\&cond $=$ cancer\&draw $=2 \&$ rank $=1$.

[12] Clinical trials-Quercitin [Internet]https://clinicaltrials.gov/ ct $2 /$ show/NCT01912820? term=quercitin\&cond $=$ cancer + prostate\&draw $=2 \&$ rank $=2$.

[13] Clinical trials-Isoquercitin [Internet]https://clinicaltrials.gov/ ct2/show/NCT02446795?term=isoquercitin\&cond=cancer + kidney\&draw $=2 \&$ rank $=1$.

[14] Clinical trials-Resveratrol-Colon cancer [Internet]https:// clinicaltrials.gov/ct $2 / \mathrm{show} / \mathrm{NCT} 00256334$ ?ter $\mathrm{m}=$ resveratrol\&cond $=$ cancer+colon \&draw $=2 \&$ rank $=1$.

[15] Clinical trials-Resveratrol-Gastrointestinal tumors [Internet]https://www.clinicaltrials.gov/ct2/show/ NCT01476592?term $=$ resveratrol\&cond $=$ cancer \&draw $=$ 2\&rank=4.

[16] Clinical trials-Polyphenols [Internet],https://www .clinicaltrials.gov/ct2/show/NCT03482401?term=phenolics + polyphenols\&cond $=$ breast + cancer $\& d r a w=2 \&$ rank $=1$.

[17] V. Uivarosi and A. C. Munteanu, "Flavonoids complexes as promising anticancer metallodrugs: From Biosynthesis to Human Health," IntechOpen, vol. 14, 2017https://www intechopen.com/books/flavonoids-frombiosynthesis-tohuman-health/flavonoid-complexes-as-promisinganticancer-metallodrugs.

[18] USDA Database for the Flavonoid Content of Selected Foods, U.S. Department of Agriculture, Agricultural Research Service [Internet], Release 3.0. Nutrient Data, 2011, http://www.ars .usda.gov/nutrientdata/flav.

[19] M. Wang, J. Firrman, L. S. Liu, and K. Yam, “A review on flavonoid apigenin: dietary intake, ADME, antimicrobial effects and interactions with human gut microbiota," BioMed Research International, vol. 2019, Article ID 7010467, 18 pages, 2019.

[20] K. Zhang, W. Song, D. Li, and X. Jin, "Apigenin in the regulation of cholesterol metabolism and protection of blood vessels," Experimental and Therapeutic Medicine, vol. 13, no. 5, pp. 1719-1724, 2017.

[21] C. Buwa, U. Mahajan, C. Patil, and S. Goyal, "Apigenin attenuates $\beta$-receptor-stimulated myocardial injury via safeguarding cardiac functions and escalation of antioxidant defence system," Cardiovascular Toxicology, vol. 16, no. 3, pp. 286297, 2016.

[22] B. Salehi, A. Venditti, M. Sharifi-Rad et al., "The therapeutic potential of apigenin," International Journal of Molecular Sciences, vol. 20, no. 6, p. 1305, 2019.

[23] X. Zhou, F. Wang, R. Zhou, X. Song, and M. Xie, "Apigenin: a current review on its beneficial biological activities," Journal of Food Biochemistry, vol. 41, no. 4, article e12376, 2017.
[24] I. Vrhovac Madunić, J. Madunić, M. Antunović et al., “Apigenin, a dietary flavonoid, induces apoptosis, DNA damage, and oxidative stress in human breast cancer MCF-7 and MDA MB-231 cells," Naunyn Schmiedebergs Archives of Pharmacology, vol. 391, no. 5, pp. 537-550, 2018.

[25] Y. J. Seo, B. S. Kim, S. Y. Chun, Y. K. Park, K. S. Kang, and T. G. Kwon, "Apoptotic effects of genistein, biochanin-A and apigenin on LNCaP and PC-3 cells by p21 through transcriptional inhibition of polo-like kinase-1," Journal of Korean Medical Science, vol. 11, pp. 1489-1494, 2011.

[26] H. Bai, H. Jin, F. Yang, H. Zhu, and J. Cai, "Apigenin induced MCF-7 cell apoptosis-associated reactive oxygen species," Scanning, vol. 36, 631 pages, 2014.

[27] F. Lindenmeyer, H. Li, S. Menashi, C. Soria, and H. Lu, “Apigenin acts on the tumor cell invasion process and regulates protease production," Nutrition and Cancer, vol. 39, no. 1, pp. 139-147, 2001.

[28] M. M. Liu, R. H. Ma, Z. J. Ni et al., “Apigenin 7-O-glucoside promotes cell apoptosis through the PTEN/PI3K/AKT pathway and inhibits cell migration in cervical cancer Hela cells," Food and Chemical Toxicology: An International Journal Published for the British Industrial Biological Research Association, vol. 146, p. 111843, 2020.

[29] X. Chen, H. Xu, X. Yu, X. Wang, X. Zhu, and X. Xu, “Apigenin inhibits in vitro and in vivo tumorigenesis in cisplatinresistant colon cancer cells by inducing autophagy, programmed cell death and targeting m-TOR/PI3K/Akt signalling pathway," Journal of B.U.O N.: official journal of the Balkan Union of Oncology, vol. 24, no. 2, pp. 488-493, 2019.

[30] A. R. Nunes, I. G. P. Vieira, D. B. Queiroz et al., "Use of flavonoids and cinnamates, the main photoprotectors with natural origin," Advances in Pharmacological and Pharmaceutical Sciences, vol. 2018, Article ID 5341487, 9 pages, 2018.

[31] L. Xu, Y. Zhang, K. Tian et al., "Apigenin suppresses PD-L1 expression in melanoma and host dendritic cells to elicit synergistic therapeutic effects," Journal of Experimental \& Clinical Cancer Research, vol. 37, no. 1, pp. 261-261, 2018.

[32] G. Zhao, X. Han, W. Cheng et al., “Apigenin inhibits proliferation and invasion, and induces apoptosis and cell cycle arrest in human melanoma cells," Oncology Reports, vol. 37, no. 4, pp. 2277-2285, 2017.

[33] S. Das, J. Das, A. Samadder, N. Boujedaini, and A. R. KhudaBukhsh, "Apigenin-induced apoptosis in A375 and A549 cells through selective action and dysfunction of mitochondria," Experimental Biology and Medicine, vol. 237, no. 12, pp. 1433-1448, 2012.

[34] L. P. Chan, T. H. Chou, H. Y. Ding et al., "Apigenin induces apoptosis via tumor necrosis factor receptor- and Bcl-2mediated pathway and enhances susceptibility of head and neck squamous cell carcinoma to 5-fluorouracil and cisplatin," Biochimica et Biophysica Acta, vol. 1820, no. 7, pp. 1081-1091, 2012.

[35] D. Kashyap, A. Sharma, H. S. Tuli et al., "Apigenin: a natural bioactive flavone-type molecule with promising therapeutic function," Journal of Functional Foods, vol. 48, pp. 457-471, 2018.

[36] N. N. Bouzaiene, F. Chaabane, A. Sassi, L. Chekir-Ghedira, and K. Ghedira, "Effect of apigenin-7-glucoside, genkwanin and naringenin on tyrosinase activity and melanin synthesis in B16F10 melanoma cells," Life Sciences, vol. 144, pp. 80-85, 2016. 
[37] A. Ghițu, A. Schwiebs, H. H. Radeke et al., “A comprehensive assessment of apigenin as an antiproliferative, proapoptotic, antiangiogenic and immunomodulatory phytocompound," Nutrients, vol. 11, no. 4, p. 858, 2019.

[38] H. H. Cao, J. H. Chu, H. Y. Kwan et al., "Inhibition of the STAT3 signaling pathway contributes to apigenin-mediated anti-metastatic effect in melanoma," Scientific Reports, vol. 6, no. 1, article 21731, 2016.

[39] C. Danciu, C. Soica, D. Antal et al., "Natural compounds in the chemoprevention of malignant melanoma," Anti-Cancer Agents in Medicinal Chemistry, vol. 18, no. 5, pp. 631-644, 2018.

[40] A. R. Nunes, Í. G. P. Vieira, D. B. Queiroz et al., "Evaluation of the cytotoxic and antiangiogenic potential of flavone apigenin using the B164A5 mouse melanoma cell line," Medicine in Evolution, vol. 25, pp. 288-295, 2020.

[41] A. W. K. Yeung, E. B. Souto, A. Durazzo et al., "Big impact of nanoparticles: analysis of the most cited nanopharmaceuticals and nanonutraceuticals research," Current research in Biotechnology, vol. 2, pp. 56-63, 2020.

[42] B. Mukherjee, S. Banerjee, and L. Mondal, "Bioactive flavonoid apigenin and its nanoformulations: a promising hope for diabetes and cancer," in Nanomedicine for Bioactives, pp. 367382, Springer, Singapore, 2020.

[43] E. A. Moacă, I. Z. Pavel, C. Danciu et al., "Romanian wormwood (Artemisia absinthium L.): physicochemical and nutraceutical screening," Molecules, vol. 24, no. 17, p. 3087, 2019.

[44] D. Ribatti, A. Vacca, L. Roncali, and F. Dammacco, "The chick embryo chorioallantoic membrane as a model for in vivo research on anti-angiogenesis," Current Pharmaceutical Biotechnology, vol. 1, no. 1, pp. 73-82, 2000.

[45] S. Avram, R. Ghiulai, I. Z. Pavel et al., Phytocompounds targeting cancer angiogenesis using the chorioallantoic membrane assay-natural products and cancer drug discovery, IntechOpen, 2017.

[46] P. Sen, P. K. Sahu, R. Haldar, K. K. Sahu, P. Prasad, and A. Roy, "Apigenin naturally occurring flavonoids: occurrence and bioactivity," UK Journal of Pharmaceutical and Bioscience, vol. 4, no. 6, pp. 56-58, 2017.

[47] S. Shukla and S. Gupta, "Apigenin: a promising molecule for cancer prevention," Pharmaceutical Research, vol. 27, no. 6, pp. 962-978, 2010.

[48] J. S. Woo, G. S. Choo, E. S. Yoo et al., “Apigenin induces apoptosis by regulating Akt and MAPK pathways in human melanoma cell A375SM," Molecular Medicine Reports, vol. 22, no. 6, pp. 4877-4889, 2020.

[49] M. A. Hasnat, M. Pervin, J. H. Lim, and B. O. Lim, “Apigenin attenuates melanoma cell migration by inducing anoikis through integrin and focal adhesion kinase inhibition," Molecules, vol. 20, no. 12, pp. 21157-21166, 2015.

[50] S. Caltagirone, C. Rossi, A. Poggi et al., "Flavonoids apigenin and quercetin inhibit melanoma growth and metastatic potential," International Journal of Cancer, vol. 87, no. 4, pp. 595$600,2000$.

[51] C. Engelmann, E. Blot, Y. Panis et al., "Apigenin - strong cytostatic and anti-angiogenic action in vitro contrasted by lack of efficacy in vivo," Phytomedicine, vol. 9, no. 6, pp. 489-495, 2002.

[52] A. A. Mrazek, L. J. Porro, V. Bhatia et al., "Apigenin inhibits pancreatic stellate cell activity in pancreatitis," The Journal of Surgical Research, vol. 196, no. 1, pp. 8-16, 2015.
[53] G. Li, C. W. Chi, X. F. Shao, and C. H. Fang, "Application of molecular imaging technology in evaluating the inhibiting effect of apigenin in vivo on subcutaneous hepatocellular carcinoma," Biochemical and Biophysical Research Communications, vol. 487, no. 1, pp. 122-127, 2017.

[54] S. Meng, Y. Zhu, J. F. Li et al., "Apigenin inhibits renal cell carcinoma cell proliferation," Oncotarget, vol. 8, no. 12, pp. 19834-19842, 2017.

[55] J. Dai, P. G. van Wie, L. Y. Fai et al., "Downregulation of NEDD9 by apigenin suppresses migration, invasion, and metastasis of colorectal cancer cells," Toxicology and Applied Pharmacology, vol. 311, pp. 106-112, 2016.

[56] X. W. Hu, D. Meng, and J. Fang, "Apigenin inhibited migration and invasion of human ovarian cancer A2780 cells through focal adhesion kinase," Carcinogenesis, vol. 29, no. 12, pp. 2369-2376, 2008.

[57] T. H. Tseng, M. H. Chien, W. L. Lin et al., "Inhibition of MDAMB-231 breast cancer cell proliferation and tumor growth by apigenin through induction of G2/M arrest and histone $\mathrm{H} 3$ acetylation-mediated p21WAF1/CIP1expression," Environmental Toxicology, vol. 32, no. 2, pp. 434-444, 2017.

[58] Q. R. WANG, X. Q. YAO, G. WEN et al., “Apigenin suppresses the growth of colorectal cancer xenografts via phosphorylation and up-regulated FADD expression," Oncology Letters, vol. 2, no. 1, pp. 43-47, 2011.

[59] L. A. Sena and N. S. Chandel, "Physiological roles of mitochondrial reactive oxygen species," Molecular Cell, vol. 48, no. 2, pp. 158-167, 2012.

[60] S. Elmore, "Apoptosis: a review of programmed cell death," Toxicologic Pathology, vol. 35, no. 4, pp. 495-516, 2007.

[61] G. Y. Liou and P. Storz, "Reactive oxygen species in cancer," Free Radical Research, vol. 44, no. 5, pp. 479-496, 2010.

[62] X. Dai, J. Zhang, F. Arfuso et al., "Targeting TNF-related apoptosis-inducing ligand (TRAIL) receptor by natural products as a potential therapeutic approach for cancer therapy," Experimental Biology and Medicine, vol. 240, no. 6, pp. 760773, 2015.

[63] C. H. Kang, I. M. N. Molagoda, Y. H. Choi, C. Park, D. O. Moon, and G. Y. Kim, "Apigenin promotes TRAIL-mediated apoptosis regardless of ROS generation," Food and Chemical Toxicology, vol. 111, pp. 623-630, 2018.

[64] S. Shukla and S. Gupta, "Apigenin-induced prostate cancer cell death is initiated by reactive oxygen species and p53 activation," Free Radical Biology and Medicine, vol. 44, no. 10, pp. 1833-1845, 2008.

[65] H. S. Seo, J. M. Ku, H. S. Choi et al., "Induction of caspasedependent apoptosis by apigenin by inhibiting STAT3 signaling in HER2-overexpressing MDA-MB-453 breast cancer cells," Anticancer Research, vol. 34, no. 6, pp. 2869-2882, 2014.

[66] X. Cao, B. Liu, W. Cao et al., "Autophagy inhibition enhances apigenin-induced apoptosis in human breast cancer cells," Chinese Journal of Cancer Research, vol. 25, no. 2, pp. 212 222, 2013.

[67] M. D. Shi, C. K. Shiao, Y. C. Lee, and Y. W. Shih, "Apigenin, a dietary flavonoid, inhibits proliferation of human bladder cancer T-24 cells via blocking cell cycle progression and inducing apoptosis," Cancer Cell International, vol. 15, no. 1, 2015.

[68] S. Shukla, E. Shankar, P. Fu, G. T. MacLennan, and S. Gupta, "Suppression of NF- $\kappa \mathrm{B}$ and NF- $\kappa \mathrm{B}$-regulated gene expression by apigenin through $\mathrm{I} \kappa \mathrm{B} \alpha$ and IKK pathway in TRAMP mice," PLoS One, vol. 10, no. 9, article e0138710, 2015. 
[69] Y. LEE, B. SUNG, Y. J. KANG et al., “Apigenin-induced apoptosis is enhanced by inhibition of autophagy formation in HCT116 human colon cancer cells," International Journal of Oncology, vol. 44, no. 5, pp. 1599-1606, 2014.

[70] E. Shankar, A. Goel, K. Gupta, and S. Gupta, "Plant flavone apigenin: an emerging anticancer agent," Current Pharmacology Reports, vol. 3, no. 6, pp. 423-446, 2017.

[71] J. Fang, Q. Zhou, L. Z. Liu et al., “Apigenin inhibits tumor angiogenesis through decreasing HIF-1alpha and VEGF expression," Carcinogenesis, vol. 28, no. 4, pp. 858-864, 2007.

[72] H. J. Kim and M. R. Ahn, "Apigenin suppresses angiogenesis by inhibiting tube formation and inducing apoptosis," Natural Product Communications, vol. 11, no. 10, pp. 1433-1436, 2016. 